\title{
Protecting and promoting systems for essential health services during rollout of COVID-19 tools
}

Kerry A. Millington

Liverpool School of Tropical Medicine

18 May 2021

\section{Question}

How to protect and ideally advance systems for essential health services whilst rolling out the COVID-19 vaccines, diagnostics and therapeutics?

- What are the opportunities COVID-19 vaccine roll out has to strengthen systems to deliver essential services?

- What are the mitigation measures needed to ensure the vaccine roll out does not displace political space and resources (finance and staff) for essential services?

\section{Contents}

1. Summary

2. Indirect impact of COVID-19 on essential health services

3. Rollout of COVID-19 tools

4. Protecting essential health services during rollout of COVID-19 tools

5. Promoting essential health services during rollout of COVID-19 tools

6. References

The K4D helpdesk service provides brief summaries of current research, evidence, and lessons learned. Helpdesk reports are not rigorous or systematic reviews; they are intended to provide an introduction to the most important evidence related to a research question. They draw on a rapid deskbased review of published literature and consultation with subject specialists. 


\section{Summary}

COVID-19 has significantly disrupted, suspended and diverted resources from essential health services which could cause a secondary health crisis and reverse decades of progress in global health. Health systems have been stretched, and in some cases collapsed, responding to the pandemic and will be stretched further with deployment of COVID-19 vaccines. Innovative adaptive measures have mitigated some of the impact of COVID-19 and presented opportunities for integrated service delivery such as testing for COVID-19 and other diseases. Countries are preparing to deploy COVID-19 vaccines at scale but the emphasis on speed and expediency over deliberate system-strengthening is coming at a cost of missing out on the benefits of long-lasting improvement that a systems approach could bring. Investment in effective and efficient service delivery for COVID-19 and essential health services will not only support the response to and recovery from COVID-19 but also strengthen the resilience of health systems to future challenges.

This helpdesk report highlights a selection of resources focusing on protecting essential health services and opportunities for strengthening during rollout of COVID-19 vaccines, testing and treatments. A search was undertaken of leading actors in this space including global health initiatives (Global Fund, Gavi, Global Financing Facility (GFF), GPEI), the United Nations agencies including WHO and UNICEF, policy think tanks (Center for Global Development (CGD), Overseas Development Institute (ODI)) amongst others. Resources include blogs, policy papers, statements, country case studies, guidelines, reports and peer reviewed research reflecting discourse, mitigation measures and any evidence of impact to-date. The resources included are not an exhaustive list; they represent those that could be identified within the time allocated. Note that specific reference to mitigation measures and strengthening of essential health services during rollout of COVID-19 vaccines are limited and are generally considered within the wider context of the response and recovery to the COVID-19 pandemic and pandemic preparedness.

A WHO Strategic Technical Advisory Group of Experts notes at this stage a key limitation of the available evidence is the lack of outcome data, particularly with control groups, that would be needed to fully evaluate the effectiveness of protection and recovery efforts (WHO, 2020b). Case studies are presented throughout the literature. There are opportunities to generate more and better evidence on what works to protect and promote more resilient health service provision and access during the response to COVID-19 (Krubiner et al., 2021).

The literature discusses adaptive and innovative measures that essential health services have taken to maintain and strengthen essential health service delivery in response to the COVID-19 pandemic and in the recovery phase. These include changes in the management of health workers and community health workers and changes in delivery of health services. Opportunities present and lessons can be learned from successful adaptive approaches to COVID-19 to protect and promote essential health services as part of the COVID-19 response and recovery efforts (Global Financing Facility, 2021b). This includes reimaging service delivery to be more effective and efficient reducing costs and improving access for the most vulnerable to reclaim the gains and accelerate progress for better health outcomes. Coordination with universal health coverage efforts will enable integrated service delivery and effective deployment of COVID-19 tools (Jakab et al., 2021). The capacity of existing health systems will need to be increased to prevent disruption, draining or redirecting of limited resources to the pandemic response. 
In March 2020, policies and public health efforts had not addressed the gendered impacts of COVID-19. Since then, the gendered dimensions of the response to and recovery from the COVID19 pandemic is being continually documented, assessed and analysed to inform and improve global and national policy responses by newly developed Working Groups and Initiatives such as the Gender and COVID-19 Working Group and The COVID-19 Gender and Development Initiative. Attention also needs to be given to the additional vulnerabilities experienced by marginalised social groups, including people living with disabilities.

\section{Indirect impact of COVID-19 on essential health services}

COVID-19 has suspended, disrupted and made inaccessible the provision of essential health services which could result in a secondary health crisis and reverse decades of progress in global health. We know from previous outbreaks and crises (Ebola, SARS, H1N1 outbreaks and the global financial crisis), and the indirect impacts of COVID-19 already observed, that indirect health effects are significant and could outweigh direct health impact (Chi et al., 2020). A successful COVID-19 policy should not be measured solely by the number of COVID-19 deaths averted but take a whole of health perspective.

In January 2021 the WHO published its interim guidance on analysing and using routine data to monitor the effects of COVID-19 on essential health services (WHO, 2021a). This guide supports interim guidance published in June 2020 on Maintaining essential health services: operational guidance for the COVID-19 context, which provides an integrated framework to guide countries in their efforts to reorganise, adapt and maintain safe delivery of high-priority essential health services within the context of the pandemic (WHO, 2020d). The Global Financing Facility (GFF) also have a guidance note on the role of country platforms in maintaining essential services and delivering on the investment case in the time of COVID-19 (Global Financing Facility, 2020).

Global efforts have examined if and to what extent essential health services have been disrupted so far during the COVID-19 pandemic. A second round of a WHO pulse survey during January to March 2021 on the continuity of essential health services during the COVID-19 pandemic reports global findings from the 135 countries and territories that participated in the key informant survey. Despite some evidence of essential health services restoration, over one year into the COVID-19 pandemic substantial disruptions persist, with about $90 \%$ of countries still reporting one or more disruptions to essential health services (WHO, 2021c). There is no substantial change since the first survey conducted in the summer of 2020, however, within countries the magnitude and extent of disruptions has generally decreased. Gains and partial rebound in service provision is likely due to implementation of recommended policies and strategies for maintaining essential health services.

In 2020, countries reported that, on average, about half of essential health services were disrupted compared to just over one third of services now being disrupted in the first three months of 2021 (WHO, 2021c). Overall, primary care and rehabilitative, palliative and long-term care are more predominantly affected. Substantial disruptions span across all major health areas. Most frequently (reported in more than $40 \%$ of countries) disrupted services are for mental, neurological and substance use disorders and neglected tropical diseases. Services across other health areas are also disrupted in more than a third of countries. Service disruptions are perceived to be caused by a mix of supply and demand side factors. Health workforce-related issues are among the most commonly reported supply-side reasons linked to staff deployment to COVID-19 relief and 
insufficient staff availability. Cancellation of elective care and changes to treatments policies are also reported. Most common demand-side reasons included: community fear/mistrust, decreases in OPD volume due to patients not presenting, travel restrictions and financial difficulties during the pandemic. In some countries, COVID-19 control may be contributing to increased barriers to accessing care (e.g., fear of getting infected, limitations in movement) and supply chain systems are also disrupted in nearly a third of countries. Many countries are using telehealth technologies to mitigate disruptions but limited access and technical capacities to use telehealth technologies are the most commonly reported barriers to use in countries. Further information, including from subnational, health facility and community levels should supplement findings to better quantify potential impact of disruptions over the short, medium and long term.

Data from ongoing GFF-supported country monitoring has reaffirmed disruptions on the supply and demand-side of essential health services in low- and lower-middle income countries from which lessons can be learned to protect and promote essential health services as part of the COVID-19 response and recovery efforts (Global Financing Facility, 2021b). Rollout of COVID-19 vaccines at scale are likely to place even more strain on health systems and resources. Impact on health financing - the ability to deliver essential health services and the ability of households to pay for these services - is likely to be significant in many of the poorest countries. This will require 'developing and implementing more effective and efficient models of delivering health care that will reduce costs and improve access for the poorest and more vulnerable populations' (Global Financing Facility, 2021b).

The Center for Global Development (CGD) present a framework for identifying the sources of indirect health effects of COVID-19 and provide a simple structure for analysts to calculate the overall "net health impact" of the pandemic (Chi et al., 2020). Sources of health system impact funding, supply chain, health workforce, infrastructure, service provision, patient access, and 'covidisation'; diseases that could be affected; most disadvantaged populations affected; and short, medium and long-term risk if not mitigated - are categorised as a subset to the overall framework. CGD also have an open access inventory of resources on indirect health impacts that is continually being updated.

A Global Fund report from 502 health facilities across Africa and Asia provides a snapshot on the impact of COVID-19 on HIV, tuberculosis (TB) and malaria services and systems for health (The Global Fund, 2021b). Disruptions on the supply-side include vulnerabilities in infection prevention measures, a lack of routine urgent care through employment, restrictions on movement and interrupted healthcare and on the demand side a wider reluctance to attend healthcare facilities because of fear of contracting COVID-19, along with increased stigma and discrimination, disruption in public transportation, lockdown or stay-at-home orders (The Global Fund, 2021b).

The COVID-19 pandemic has severely disrupted access to TB services and is reversing the gains made towards ending TB (WHO, 2021b). Latest data collected by WHO from more than 80 countries indicates that 1.4 million fewer people received care for TB in 2020 than in 2019, a 21\% drop in TB notifications over this period. This could lead to an additional half a million TB deaths. The consequences of COVID-19 on access to TB services and on the determinants of TB, including poverty and food security, make it critical to adopt a multisectoral approach with engagement of affected communities, and civil society to stop further declines in case notifications and get on track to end TB. Global extreme poverty will rise for the first time in over 20 years, according to the World Bank, thereby causing significant increase in undernourishment, a major 
determinant of TB. In this joint statement the WHO and CSTF-TB urge governments, partners and stakeholders to leverage domestic and external financial resources, including funding from The Global Fund and other bilateral and multilateral funding agencies, to introduce and scale-up innovative approaches for provision of TB services, including the use of digital technologies and dual testing for TB and COVID-19, and to strengthen airborne infection control, including procurement of personal protective equipment (PPE) for health workers and community workers involved in TB care and prevention activities.

An estimated 11.5 million people affected by disruptions to anti-retroviral services for HIV/AIDS in 36 countries between April and June 2020 and $75 \%$ of the Global Fund's HIV/AIDS programmes report moderate to high levels of disruption to service delivery.

A systematic review and meta-analysis found that global maternal and foetal outcomes have worsened during the COVID-19 pandemic, with an increase in maternal deaths, stillbirth, ruptured ectopic pregnancies and maternal depression (Chmielewska et al., 2021). This highlights the urgency behind prioritising safe, accessible, and equitable maternity care within the strategic response to this pandemic and in future health crises. The GFF estimates that access to life-saving health interventions for women, children and adolescents in 36 of the world's poorest countries has dropped by as much as $25 \%$ due to COVID-19. That is equivalent to 82 million children not receiving oral rehydration, 4 million women losing access to care during childbirth, and 17 million children missing out on vaccinations. This threatens to reverse gains on the health of women, children and adolescents. The GFF Case for Investment calls for $\$ 1.2$ billion funding to stop secondary health crisis for women and children due to the COVID-19 pandemic to help limit the damage and reclaim the gains (Global Financing Facility, 2021a). Gavi, WHO and UNICEF warn that at least 80 million children under the age of one risk missing out on routine vaccines for disease such as measles, polio and diphtheria (WHO, 2020c).

Background paper 8 to The Independent Panel for Pandemic Preparedness and Response main report on Impact on Essential Health Services summarises the disruptions to essential health services with a deep dive into low- and middle-income country impacts (The Independent Panel for Pandemic Preparedness and Response, 2021b). A rational approach to public sector health spending and decision making during the COVID-19 pandemic based on ethics and equity principles is crucial to ensure that patients not infected by COVID-19 continue to get access to healthcare and that the services they need continue to be resourced (Williams et al., 2021).

\section{Rollout of COVID-19 tools}

Access to the COVID-19 Tools Accelerator (ACT-A) is co-convened by nine global health organisations which supports the development and equitable distribution of tests, treatments and vaccines, and since September 2020, the strengthening of health systems through the Health Systems Connector (HSC) pillar. HSC supports the integrated delivery of COVID-19 tools, rapidly addresses bottlenecks, and manages linkages and synergies with complementary activities for the delivery of essential health services and strengthening of health systems (WHO, 2021d). Noted though that 'funds committed so far are skewed towards vaccines, with little for health systems' and primarily dedicated to the procurement of two key tools: PPE and medical oxygen with a range of issues which exist that are not covered by the $\$ 9$ billion health systems request - the cost of disposal of PPE, investments in electricity grids and pipes to make oxygen functional, the shortage of health workers, training and the need for infrastructure to support cold chains (Usher, 2021). 
HSC has developed a collection of assessment tools to help governments partners and donors generate and use real-time data from those at the frontline to facilitate the scale-up and delivery of essential COVID-19 tools, guide strategies and plans to maintain essential health services (WHO, 2021d). Kenya started using these tools at the end of 2020, interviewing facility managers and community health care workers in frontline services over one month and using the information to shape and strengthen their response to the pandemic and at the same time ensure continuity of services (WHO, 2021d).

WHO's COVID-19 Vaccine Introduction Readiness Assessment Tool provides support to countries in assessing programme readiness to introduce COVID-19 vaccines, identifying gaps and prioritising actions to be ready; and identifying opportunities for financial support. In anticipation of the availability of safe and effective vaccines for COVID-19, assessments of country readiness to deploy COVID-19 vaccines were rolled out in November 2020 jointly by the World Bank and WHO together with Gavi, the Global Vaccine Alliance; the Global Fund; UNICEF and governments (The World Bank, 2021). Initial findings from 128 countries as of March 2021 show that the world's poorest countries are at varying degrees of readiness for vaccinating their populations against COVID-19. The tremendous impact of COVID-19 on health and economies, fear of highly contagious variants and pressure to start vaccinations have prompted many countries to prepare well enough to begin inoculating their populations when vaccines are available. Large-scale vaccination mobilisation provides opportunities to countries to sustainably strengthen the cold chain and introduce environmentally friendly options that could be of use well beyond the current crisis. However, the emphasis on speed and expediency over deliberate system-strengthening is coming at a cost of missing out on the benefits of long-lasting improvement that a systems approach could bring. Few countries are using the opportunity provided by the deployment of COVID-19 vaccines to strengthen health services and health systems and find long-lasting solutions for similar future challenges. With 47 African countries now rolling out COVID-19 vaccines and over 17 million doses now given lessons are emerging from countries that have made strong progress including 'existing health systems giving a strong base' (Gavi, 2021a).

Recognising that introduction of a new vaccine can have both positive and negative impacts on a country's health system, WHO SAGE-endorsed six guiding principles for countries to follow in planning and implementing a new vaccine introduction whilst strengthening their overall immunisation programme and health system (WHO, 2020e). WHO and UNICEF recommend that 'activities should be integrated into the national primary health care (PHC) operational framework including: microplanning, using an evidence-based decision-making process to govern the introduction of the COVID-19 vaccine; strengthening human resources management; training for new vaccine introduction; establishing new contact points for vaccination across the life course; ensuring traceability systems and technologies are leveraged to ensure the integrity and efficiency of supply chains, improving and expanding integrated project management and the supply chain; enhancing integrated disease surveillance and adverse effects following immunisation monitoring and reporting systems; and conducting integrated advocacy and communications activities to promote demand for vaccination as part of increasing overall demand and acceptability of all essential PHC services' (WHO \& UNICEF, 2020).

New variants of concern threaten the effectiveness of existing COVID-19 vaccines, vaccine hesitancy slowing rollout, including in Africa, and interrupted and limited supply of COVID-19 tools (Gavi, 2021c; Vaccine Hesitancy Slows Africa's COVID-19 Inoculation Drive / Reuters, 2021). Note that reasons for refusing a COVID-19 vaccine extend beyond just hesitancy (Gavi, 2021b; Ratzan 
et al., 2021). As of 12 April, less than $2 \%$ of the 780 million COVID-19 vaccines given globally have been administered in Africa (Gavi, 2021a) and across 24 countries in Africa, only $11 \%$ of health facilities could conduct COVID-19 antigen rapid diagnostic tests, and only $8 \%$ could conduct PCR tests (The Global Fund, 2021b).

\section{Protecting essential health services during rollout of COVID-19 tools}

Adapting to the impact of COVID-19 on health service continuity with innovative approaches are enabling services to be implemented safely and mitigate negative impacts and sometimes even increase essential health service utilisation (The Global Fund, 2021b). These include the management of health and community workers and/or in changes in delivery strategies for health services unrelated to COVID-19. Such as facilities extending drug prescriptions to ensure patients have long-term and uninterrupted access to their medication; switching from a centralised approach to door-to-door distribution of long-lasting insecticidal nets (LLIN) against mosquitoes; but also, integration of services and especially for COVID-19 and TB which have much in common. Note that one size does not fit all with adaptive measures needing to be tailored to the country and local context. More funding is required though to scale up adaptive measures which are working, accelerating new approaches and innovations to improve service delivery and provide an opportunity for synergies in delivering health services, protecting frontline health workers and maintaining essential health services.

WHO operational guidance for maintaining essential health services during an outbreak provides guidance on a set of immediate actions that countries should consider at national, regional and local level to recognise, strategically plan and coordinate action to maintain essential health service delivery mitigating the risk of system collapse (WHO, 2020d). Blanchet et al make the case for a rational approach to public sector health spending and decision making during and in the early recovery phase of the COVID-19 pandemic (Blanchet et al., 2020). A list of 120 essential nonCOVID-19 health interventions is presented, adapted from the model of health benefit packages developed by the Disease Control Priorities project. Focusing on routine healthcare services, this list guides governments and humanitarian agencies working to define national and local guidance for protecting essential routine services in low-income and middle-income countries and humanitarian settings and which interventions policymakers and donors need to protect from disinvestment.

Background paper 8 to The Independent Panel for Pandemic Preparedness \& Response's main report published in May 2021 (The Independent Panel for Pandemic Preparedness and Response, 2021a) on Impact on essential health services highlights experiences of an emerging evidence base, drawn from case studies from multiple countries, of protection and recovery efforts (The Independent Panel for Pandemic Preparedness and Response, 2021b). A 2020 meeting of the WHO Strategic Technical Advisory Group of Experts note that a key limitation of the available evidence is the lack of outcome data, particularly with control groups, that would be needed to fully evaluate the effectiveness of these approaches and that support for data collection, aggregation and knowledge translation mechanisms coordinated at the global level are required to support realtime learning on disruptions and effective mitigation strategies across countries (WHO, 2020b).

The WHO issued an Information note on 5 May 2021 intended to assist national TB programmes and health personnel worldwide to maintain essential TB services during the COVID-19 pandemic 
and in the recovery phase and what services can be leveraged across both diseases including basic infection prevention and control, contact tracing and household and community-based care (WHO, 2021e). A Global Fund COVID-19 Information Note also provides guidance to countries on preparing and updating plans to mitigate the impact of COVID-19 on TB services (The Global Fund, 2020). Specifically, to identify opportunities for the response to TB to benefit from the response to COVID-19, including enhanced surveillance for both diseases and scale up of the promotion and protection of human rights and combating stigma and discrimination.

A CGD Brief provides a snapshot of the existing and evolving evidence as it relates to key health services and outcomes for women and girls in low- and middle-income countries, focusing primarily on sexual and reproductive health and rights (Krubiner et al., 2021). The paper examines some ways national governments have sought to maintain provision of essential health services and reviews the extent to which donor institutions have prioritised financial, technical and other forms of support to mitigate disruptions. Areas of opportunity for future action that donors and governments should pursue to address indirect harms to women's and girl's health during and beyond the COVID-19 crisis include improving data collection and sharing, with explicit attention to gender and intersectional disadvantage, and evaluating what works to mitigate gendered indirect effects, especially integrated approaches.

An analysis, based on a rapid evidence assessment by the Social Science in Humanitarian Action Platform, on mitigating negative impacts on other areas of health emphasises the additional vulnerabilities experienced by marginalised social groups, including people living with disabilities (Hrynick et al., 2021). In the short-term, there is a need to embed the COVID-19 response within integrated health systems approaches feeding into longer-term strategies to strengthen health systems, expand universal health care coverage and address the social determinants of health.

Attention has been given on how to prioritise between patient groups for COVID-19 vaccination and how to ensure equity but these decisions must balance the COVID-19 response with the need to invest in other healthcare (Williams et al., 2021). Here principles of good priority setting including evidence-based and transparent decision-making, participation of stakeholders and a focus on the implementation of decisions can be helpful.

\section{Promoting essential health services during rollout of COVID-19 tools}

A blog from, UHC2030 co-chairs highlights that 'just as everyone, everywhere should have access to quality, safe, effective and affordable COVID-19 tests, treatments and vaccines, they should also have access to quality and affordable health services across their health needs' (Cuevas Barron \& Koonin, 2021). In some countries, COVID-19 has hampered the ability of health systems to deliver on essential health services and even exposed a relative neglect of core public health functions - 'building better' must mean investing in health systems in a joined-up way.

A key HSC priority for 2021 is to manage linkages and synergies with complementary activities for the delivery of essential health services and strengthening of health systems to help meet the primary objective of enabling integrated delivery and effective deployment of COVID-19 tools (Jakab et al., 2021). Complementary activities are: 
- Coordination with the ongoing efforts to support a comprehensive COVID-19 response and address the secondary health crisis

- Coordination with the broader complementary health systems work, including the UHC2030 and UHC partnership

- Increase of capacity of existing health systems and prevention of disruption, draining or redirecting of limited resources to the pandemic response

Background paper 8 to The Independent Panel for Pandemic Preparedness \& Response's main report (The Independent Panel for Pandemic Preparedness and Response, 2021a) on the Impact on essential health services recommends that to minimise disruptions to essential services and protect health workers during future health emergencies to (1) better invest in, better prepare, and better protect the health workforce; (2) build the evidence-base for emerging health systems adaptations that could improve health systems resilience and pandemic response, as well as efficient service delivery in peace time; and (3) investment in foundational effective prevention systems to protect essential health services. (The Independent Panel for Pandemic Preparedness and Response, 2021b).

Most public health efforts are concentrated on improving systems' capacity to respond to the COVID-19 pandemic but tend to overlook gender-specific experiences and challenges missing an opportunity to design and implement policies and systems more effectively. Existing policies may even deepen structural inequalities. A rapid literature review by the Maintains Programme details successful interventions that could enable adaptive approaches to respond to gender concerns relevant to the COVID-19 pandemic including the concern of transactional sex for vaccines (Jhunjhunwala \& Padmanabhan, 2020).

A working paper by CGD addresses the gendered impact of COVID-19 focusing on how the pandemic has been affected women's and girl's health (Krubiner et al., 2021). The paper concludes with six opportunities and preliminary recommendations of promising strategies that donors and governments could follow to address the indirect harms to their health. This includes building on the momentum of health innovations during the COVID-19 crisis during the response and recovery phase by integrating service delivery, including new digital models and tools into the larger health systems to realise their full potential, to better meet the broader health needs of women and girls. Note that given that COVID-19 has amplified pre-existing vulnerabilities and inequities the preCOVID evidence on health systems strengthening still applies.

An influential commentary on COVID-19: The Gendered Impacts of the Outbreak published in The Lancet in March 2020 (Wenham et al., 2020) led to the creation of an international Gender and COVID-19 Working Group bringing academics together to conduct real-time gender analysis to identify and document the gendered dynamics of COVID-19 and gaps in preparedness and response. Outputs include development of a tool to describe differences in the secondary health effects of the COVID-19 pandemic across genders to help decision makers address gender disparities in pandemic preparedness, response, and recovery plans ('How to Create a Gender Responsive Pandemic Plan Addressing the Secondary Effects of COVID-19 > Gender \& Covid19', n.d.). The CGD has also set up a new global research hub: The COVID-19 Gender and Development Initiative.

A Stop TB Partnership report highlights the opportunity to leverage investments in the ACT-A to strengthen national TB and TB/COVID-19 responses, as the mechanism seeks to find and fund 
solutions and improve disease surveillance, especially in regions and countries with weak health systems (Stop TB Partnership, 2020). The commodities and tools developed for COVID-19 should stimulate the diversification of tools, including:

- The development of multiplexed point-of-care testing (xPOCT) diagnostic tools, which will support options such as bidirectional screening of COVID-19 and TB

- Increased access to scientific data through proliferation of open-source systems

- Challenging the elasticity of the current patent system

India's experience shows that the COVID-19 pandemic provides an opportunity to fight TB and COVID-19 at the same time by increasing investments in the common tools, health workers and systems for health needed for both diseases (The Global Fund, 2021a). India developed a plan to integrate TB and COVID-19, including screening programmes - screening of TB patients for COVID-19 and COVID-19 patients for TB (known as bi-directional screening) to increase surveillance and laboratory services. Officials and heath community workers say the fight against COVID-19 is now providing an opportunity to better fight TB with the expansion of molecular testing and changes in social norms. For example in Mumbai, India a new technology - Truelab ${ }^{\text {TM }}$ which is an innovative, chip-based, multi-disease diagnostic platform is being used to expand COVID-19 testing and to facilitate TB screening of people with COVID-19 symptoms (FIND and IHF Partner with MCGM to Expand Access to COVID-19 and Tuberculosis Dual Testing in Mumbai, 2021).

A WHO report documents the significant role played by polio eradication personnel during the pandemic and urges strong action to sustain this network to deliver essential public health services after polio is eradicated (WHO, 2020a). The polio programme plans to use its expertise in immunisation to help deliver COVID-19 vaccines as well as reach at least 80 million children who have missed out on vital vaccines during the pandemic. This accelerates the "polio transition" process to serve other public health goals, health emergencies and health systems strengthening whilst simultaneously providing support to countries to develop plans on how polio capabilities can be sustained (Polio Global Eradication Initiative, 2021).

\section{References}

Blanchet, K., Alwan, A., Antoine, C., Cros, M. J., Feroz, F., Amsalu Guracha, T., Haaland, O., Hailu, A., Hangoma, P., Jamison, D., Memirie, S. T., Miljeteig, I., Jan Naeem, A., Nam, S. L., Norheim, O. F., Verguet, S., Watkins, D., \& Johansson, K. A. (2020). Protecting essential health services in low-income and middle-income countries and humanitarian settings while responding to the COVID-19 pandemic. BMJ Global Health, 5(10), e003675. https://doi.org/10.1136/bmjgh-2020-003675

Chi, Y.-L., Regan, L., Nemzoff, C., \& Walker, D. (2020). Beyond COVID-19: A Whole of Health Look at Impacts During the Pandemic Response. Center for Global Development; 177. https://www.cgdev.org/publication/beyond-covid-19-whole-health-look-impacts-duringpandemic-response

Chmielewska, B., Barratt, I., Townsend, R., Kalafat, E., van der Meulen, J., Gurol-Urganci, I., O'Brien, P., Morris, E., Draycott, T., Thangaratinam, S., Le Doare, K., Ladhani, S., von Dadelszen, P., Magee, L., \& Khalil, A. (2021). Effects of the COVID-19 pandemic on 
maternal and perinatal outcomes: A systematic review and meta-analysis. The Lancet Global Health, S2214109X21000796. https://doi.org/10.1016/S2214-109X(21)00079-6

Cuevas Barron, G., \& Koonin, J. (2021, April 22). A message from UHC2030 Co-Chairs on the first anniversary of the Access to COVID-19 Tools Accelerator. UHC2030.

https://www.uhc2030.org/blog-news-events/uhc2030-news/a-message-from-uhc2030-cochairs-on-the-first-anniversary-of-the-access-to-covid-19-tools-accelerator-555472/

FIND and IHF partner with MCGM to expand access to COVID-19 and tuberculosis dual testing in Mumbai. (2021, February 17). FIND. https://www.finddx.org/newsroom/pr-17feb21/

Gavi. (2021a, May 6). Emerging lessons from Africa's COVID-19 vaccine rollout. https://www.gavi.org/vaccineswork/emerging-lessons-africas-covid-19-vaccine-rollout

Gavi. (2021b, May 10). When refusing a COVID-19 vaccine isn't about hesitancy. https://www.gavi.org/vaccineswork/when-refusing-covid-19-vaccine-isnt-about-hesitancy

Gavi. (2021c, May 12). Kenya completes its first round of COVID-19 vaccinations. https://www.gavi.org/vaccineswork/kenya-completes-its-first-round-covid-19-vaccinations

Global Financing Facility. (2020). Guidance note: The role of country platforms in maintaining essential services and delivering on the investment case in the time of COVID-19. https://www.globalfinancingfacility.org/sites/gff_new/files/documents/GFF-CountryPlatforms-Guidance-Note-Covid-19.pdf

Global Financing Facility. (2021a). Reclaim the Gains: The Case for Investing in the Global Financing Facility 2021-2025. https://www.globalfinancingfacility.org/sites/gff_new/files/documents/case-for-investmentgff.pdf

Global Financing Facility. (2021b). One Year into the Pandemic: Using Data to Protect and Promote Essential Health Services as Part of the COVID-19 Response and Recovery (Twelfth Investors Group Meeting). https://www.globalfinancingfacility.org/sites/gff_new/files/documents/GFF-IG12-3Protecting-Essential-Health-Services.pdf

How to create a gender responsive pandemic plan Addressing the secondary effects of COVID$19>$ Gender \& Covid-19. (n.d.). Gender \& Covid-19. Retrieved 17 May 2021, from https://www.genderandcovid-19.org/how-to-create-a-gender-responsive-pandemic-planaddressing-the-secondary-effects-of-covid-19/

Hrynick, T. A., Ripoll Lorenzo, S., \& Carter, S. E. (2021). COVID-19 response: Mitigating negative impacts on other areas of health. BMJ Global Health, 6(4), e004110. https://doi.org/10.1136/bmjgh-2020-004110

Jakab, Z., Pate, M., \& Sands, P. (2021). Health Systems Connector country-level implementation. https://www.who.int/publications/m/item/health-systems-connectorcountry-level-implementation

Jhunjhunwala, G., \& Padmanabhan, V. (2020). Rapid Literature Review: Gender. 25. 
Krubiner, C., O'Donnell, M., Kaufman, J., \& Bourgault, S. (2021). Addressing the COVID-19 Crisis's Indirect Health Impacts for Women and Girls. Center for Global Development, 4.

Polio Global Eradication Initiative. (2021, February 8). COVID-19 shows value of polio infrastructure to support resilient health systems. https://polioeradication.org/newspost/covid-19-shows-value-of-polio-infrastructure-to-support-resilient-health-systems/

Ratzan, S., Schneider, E. C., Hatch, H., \& Cacchione, J. (2021). Missing the Point-How Primary Care Can Overcome Covid-19 Vaccine "Hesitancy". New England Journal of Medicine, NEJMp2106137. https://doi.org/10.1056/NEJMp2106137

Stop TB Partnership. (2020). The Deadly Divide: TB Commitments vs TB Realities. http://www.stoptb.org/assets/documents/communities/The\%20Deadly\%20Divide_TB\%20 Commitments\%20vs\%20TB\%20Realities\%20FINAL\%20HLM\%20Report.pdf

The Global Fund. (2020). COVID-19 Information Note: 'Catch-up' Plans to Mitigate the Impact of COVID-19 on Tuberculosis Services.

http://www.stoptb.org/assets/documents/covid/covid19_tuberculosisservicesimpact_guid ancenote_en.pdf

The Global Fund. (2021a, March 24). Fighting Two Airborne Pandemics in India: TB and COVID19 by The Global Fund-Global Fund Special Coverage.

https://globalfund.exposure.co/fighting-two-airborne-pandemics-in-india-tb-and-covid19

The Global Fund. (2021b). The impact of COVID-19 on HIV, TB and malaria services and systems for health: A snapshot from 502 health facilities across Africa and Asia. https://www.theglobalfund.org/media/10776/covid-19_2020-disruptionimpact_report_en.pdf

The Independent Panel for Pandemic Preparedness and Response. (2021a). COVID-19: Make it the Last Pandemic. https://theindependentpanel.org/wp-content/uploads/2021/05/COVID19-Make-it-the-Last-Pandemic_final.pdf

The Independent Panel for Pandemic Preparedness and Response. (2021b). Impact on Essential Health Services Background paper 8. https://theindependentpanel.org/wpcontent/uploads/2021/05/Background-paper-8-Impact-on-Essential-Health.pdf

The World Bank. (2021). Assessing Country Readiness for COVID-19 Vaccines First Insights from the Assessment Rollout.

http://documents1.worldbank.org/curated/en/467291615997445437/pdf/AssessingCountry-Readiness-for-COVID-19-Vaccines-First-Insights-from-the-AssessmentRollout.pdf

Usher, A. D. (2021). Health systems neglected by COVID-19 donors. The Lancet, 397(10269), 83. https://doi.org/10.1016/S0140-6736(21)00029-5

Vaccine hesitancy slows Africa's COVID-19 inoculation drive / Reuters. (2021, May 4). Reuters. https://www.reuters.com/world/africa/vaccine-hesitancy-slows-africas-covid-19inoculation-drive-2021-05-04/ 
Wenham, C., Smith, J., \& Morgan, R. (2020). COVID-19: The gendered impacts of the outbreak. The Lancet, 395(10227), 846-848. https://doi.org/10.1016/S0140-6736(20)30526-2

WHO. (2020a). Contributions of the polio network to COVID-19 response: Turning the challenge into an opportunity for polio transition (p. 35).

WHO. (2020b). Meeting of the WHO Strategic and Technical Advisory Group of Experts (STAGE).

WHO. (2020c, May 22). At least 80 million children under one at risk of diseases such as diphtheria, measles and polio as COVID-19 disrupts routine vaccination efforts, warn Gavi, WHO and UNICEF. https://www.who.int/news/item/22-05-2020-at-least-80-millionchildren-under-one-at-risk-of-diseases-such-as-diphtheria-measles-and-polio-as-covid19-disrupts-routine-vaccination-efforts-warn-gavi-who-and-unicef

WHO. (2020d). Maintaining essential health services: Operational guidance for the COVID-19 context interim guidance. https://www.who.int/publications-detail-redirect/WHO-2019nCoV-essential-health-services-2020.1

WHO. (2020e). DRAFT Immunization as an Essential Health Service: Guiding principles for immunization activities during times of severe disruption, incluing during the COVID-19 pandemic.

https://www.who.int/immunization/sage/meetings/2020/october/Session02A_GuidingPrin ciplesImmunizationServices.pdf

WHO. (2021a). Analysing and using routine data to monitor the effects of COVID-19 on essential health services: Practical guide for national and subnational decision-makers. https://www.who.int/publications-detail-redirect/who-2019-nCoV-essential-healthservices-monitoring-2021-1

WHO. (2021b). Joint Statement: WHO Director-General and the Civil Society Task Force on TB Urgent actions to stop preventable deaths and suffering due to tuberculosis and recover gains lost during the COVID-19 pandemic. https://mcusercontent.com/f093a7c38a3780cd9504f8d9d/files/a5be833a-b73d-47b6961c-dcd865413e60/CSTF_DG_Statement_1_.pdf

WHO. (2021c). Second round of the national pulse survey on continuity of essential health services during the COVID-19 pandemic January-March 2021.

WHO. (2021d). Act now, ACT together 2020-2021 Impact Report. https://www.who.int/publications/m/item/act-now-act-together-2020-2021-impact-report

WHO. (2021e). WHO Information Note: COVID-19 considerations for tuberculosis (TB) care. https://www.who.int/publications/i/item/WHO-2019-nCoV-TB-care-2021.1

WHO, \& UNICEF. (2020). Guidance on developing a national deployment and vaccination plan for COVID-19 vaccines. https://apps. who.int/iris/bitstream/handle/10665/336603/WHO2019-nCoV-Vaccine_deployment-2020.1-eng.pdf

Williams, I., Essue, B., Nouvet, E., Sandman, L., Razavi, S. D., Noorulhuda, M., Goold, S., Danis, M., Biemba, G., Abelson, J., \& Kapiriri, L. (2021). Priority setting during the 
COVID-19 pandemic: Going beyond vaccines. BMJ Global Health, 6(1), e004686. https://doi.org/10.1136/bmjgh-2020-004686

\section{Suggested citation}

Millington, K.A. (2021). Protecting and promoting systems for essential health services during rollout of COVID19 tools. K4D Helpdesk Report. Institute of Development Studies. DOI: 10.19088/K4D.2021.084

\section{About this report}

This report is based on six days of desk-based research. The K4D research helpdesk provides rapid syntheses of a selection of recent relevant literature and international expert thinking in response to specific questions relating to international development. For any enquiries, contact helpdesk@k4d.info.

K4D services are provided by a consortium of leading organisations working in international development, led by the Institute of Development Studies (IDS), with the Education Development Trust, Itad, University of Leeds Nuffield Centre for International Health and Development, Liverpool School of Tropical Medicine (LSTM), University of Birmingham International Development Department (IDD) and the University of Manchester Humanitarian and Conflict Response Institute (HCRI).

This report was prepared for the UK Government's Foreign, Commonwealth \& Development Office (FCDO) and its partners in support of pro-poor programmes. Except where otherwise stated, it is licensed for non-commercial purposes under the terms of the Open Government Licence v3.0. K4D cannot be held responsible for errors or any consequences arising from the use of information contained in this report. Any views and opinions expressed do not necessarily reflect those of FCDO, K4D or any other contributing organisation.

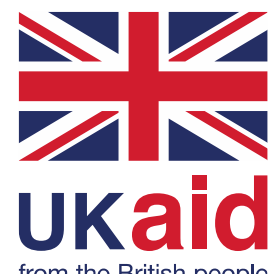

(C) Crown copyright 2021 